\title{
Carers' education improves oral health of older people suffering from dementia - results of an intervention study
}

This article was published in the following Dove Press journal:

Clinical Interventions in Aging

30 November 2016

Number of times this article has been viewed

\author{
Andreas Zenthöfer' \\ Inga Meyer-Kühling 2,3 \\ Anna-Luisa Hufeland' \\ Johannes Schröder ${ }^{2,3}$ \\ Tomas Cabrera' \\ Dominik Baumgart \\ Peter Rammelsberg' \\ Alexander J Hassel' \\ 'Department of Prosthetic Dentistry, \\ University of Heidelberg, ${ }^{2}$ Institute of \\ Gerontology, University of Heidelberg, \\ ${ }^{3}$ Section of Geriatric Psychiatry, \\ Center of Psychiatry, University of \\ Heidelberg, Heidelberg, Germany
}

Aim: The aim of this study was to evaluate the effectiveness of carers' education on improvements in oral health and denture hygiene of care-dependent and cognitively impaired older people in nursing homes compared to those without intervention.

Methods: A total of 219 seniors living in 14 nursing homes in southwest Germany (intervention: $n=144$; control: $n=75$ ) were enrolled in this study. For each participant, Plaque Control Record (PCR), Gingival Bleeding Index (GBI), Denture Hygiene Index (DHI) and Community Periodontal Index of Treatment Needs (CPITN) were assessed at baseline and six months following the interventions. In addition, demographic parameters such as age, sex, chronic diseases, permanent medications, level of dependency and cognitive state were recorded. In the intervention homes, education for caregivers was provided and ultrasound baths for denture cleaning were implemented. Changes in the dental target variables PCR, GBI, CPITN and DHI during the six-month study period were compared between subjects in the intervention and the control groups as well as between subjects with and without dementia. Additionally, multivariate models were compiled for each dental index to evaluate possible confounders.

Results: In the intervention group, PCR and DHI significantly improved during the study period $(P<0.001)$. Oral health and denture hygiene improved likewise in subjects with and without dementia. In the control group, no significant improvements were observed $(P>0.05)$.

Conclusion: Carers' education improves oral health of people in nursing homes over a clinically relevant period of time. Implementation of ultrasound baths is a simple and effective measure to improve denture hygiene of both institutionalized elderly people and seniors with dementia and in severe need of care. From a clinical standpoint, it is noteworthy that the respective interventions can be easily implemented in everyday care routine.

Keywords: oral health, intervention, older people, nursing home, longitudinal

\section{Introduction}

The demographic change leads to a larger proportion of older people in many countries. In Europe, for example, the median age increased from 36 years in 1992 to 42 years in 2016. Subsequently, this development entails more people in need of care. The literature reflects many oral problems of older people in need of care, ${ }^{1,2}$ while oral health of nursing home residents is considered even more compromised..$^{3-5} \mathrm{~A}$ variety of factors seem to produce an increased likelihood of deterioration of oral health conditions. On the one hand, both polypharmacia and multimorbidity - which are highly prevalent in nursing home residents - can contribute to oral problems; on the other hand, the vast majority of older people in need of residential care show cognitive
Correspondence: Andreas Zenthöfer Department of Prosthetic Dentistry, University of Heidelberg, Im

Neuenheimer Feld 400, 69120

Heidelberg, Germany

Tel +49 622I 567332

Fax +49 622I 56 537I

Email andreas.zenthoefer@med.

uni-heidelberg.de 
and motor disabilities that hinder adequate oral hygiene and access to dental health care provision. At this stage, carers have a key position in maintaining older peoples' oral health. ${ }^{6-8}$ Nonetheless, carers have to deal with many other tasks such as washing, dressing, toileting and coordinating physicians' visits with the result that oral care often has no priority. These problems, however, are emphasized by the fact that nurses miss knowledge on the specific oral hygiene requirements of older people. ${ }^{6,7} \mathrm{~A}$ further limitation of adequate oral hygiene provision is care-resistant behavior, which can be frequently observed in people suffering from dementia. ${ }^{9}$ Previous studies indicated poor oral hygiene in subjects with dementia in comparison to those without cognitive impairments, namely, more caries increments and worse periodontal conditions, ${ }^{10-13}$ and the prevalence of cognitive and motor impairments in nursing homes is likewise high ( $\sim 50 \%$ of institutionalized elderly). However, maintaining a lifelong good oral health is important for both oral function (nutrition) and its impact on general health such as cardiovascular diseases, stroke, diabetes mellitus and pneumonia. ${ }^{14-16}$ Some authors made an attempt to improve oral conditions of nursing home residents, mainly with the focus on relatively autonomous older people. It has been demonstrated that residents' education in teeth brushing techniques has a positive effect on oral hygiene. Further, studies have investigated the effects of carers' education and found improved oral hygiene, less gingivitis, a reduced prevalence of denture stomatitis and improved denture hygiene. ${ }^{17-19}$ Additionally, some studies stressed the positive effects of professional teeth cleaning leading to a reduction in oral pathogens such as Candida and prevalence of febrile diseases and pneumonia; the same was true for reduction in denture plaque. ${ }^{20}$ However, the literature lacks reports on the effectiveness of carers' education in more compromised nursing home residents in need of intensive care and/or suffering from dementia. Sparse available literature indicates only marginal improvements in oral hygiene as well as denture hygiene after implemented interventions. ${ }^{21,22}$ However, it remains unclear if carers' teaching on gerodontologic issues is capable of improving oral health of demented and care-dependent older people over a clinically relevant period.

This study, therefore, aimed to evaluate the effectiveness of carers' education and implementation of ultrasound baths for denture cleaning on the oral health and denture hygiene of cognitively and motorically impaired older people after six months following the interventions, and to compare the outcome to a control group without interventions.

\section{Methods}

\section{Sample}

This controlled trial was part of an interdisciplinary research project that was initiated and supported by the Federal Ministry of Social Affairs, Baden-Württemberg, Germany. The study deals with questions concerning medical services for residents of nursing homes, their quality of life and the improvement in both. The investigation procedures were approved by the local ethics committee of the University of Heidelberg (approval no S-002/2012). Fourteen nursing homes in southwest Germany were selected to be representative by the Federal Ministry of Social Affairs of the state Baden-Württemberg. No participant-related allocation to intervention or control group was performed by the research team because of possible side effects of the interventions in a nursing home on participants in the control group. The Federal Ministry of Social Affairs assigned the nursing homes instead at random to intervention and control homes (eight intervention homes/six control homes). The inclusion criteria for participation of the residents were that they had natural remaining teeth and/or dentures and did not plan moving home during the study period. It was further demanded that participants or their legal guardians - in case they were not sui legis (incapable of giving informed consent by law) - signed an informed consent document. No further inclusion criteria were used in order to examine a preferably wide spectrum of seniors. Two-hundred and seventy-seven residents agreed to participate and were included in the study. Cross-sectional characteristics are published elsewhere. ${ }^{13}$

\section{Study sequence}

A detailed study flowchart according to CONSORT criteria can be seen in Figure 1. Prior to the interventions for the carers, each participant underwent a comprehensive dental examination and an assessment of cognitive state (baseline visit). The dental examinations were repeated after six months following the intervention (follow-up visit). The dental examinations were carried out by two dentists, experienced in epidemiologic surveys. In terms of validity, they were calibrated at the Department of Prosthodontics, University of Heidelberg, and tested for their interrater agreement by double investigations of 15 participants of the sample. Agreement for the administered findings was Cronbach's $\alpha>0.9$. The examiners were blinded against the group membership (intervention/control group), and both performed dental examinations of participants in all 14 nursing homes according to their time schedule. The second examiner did not 


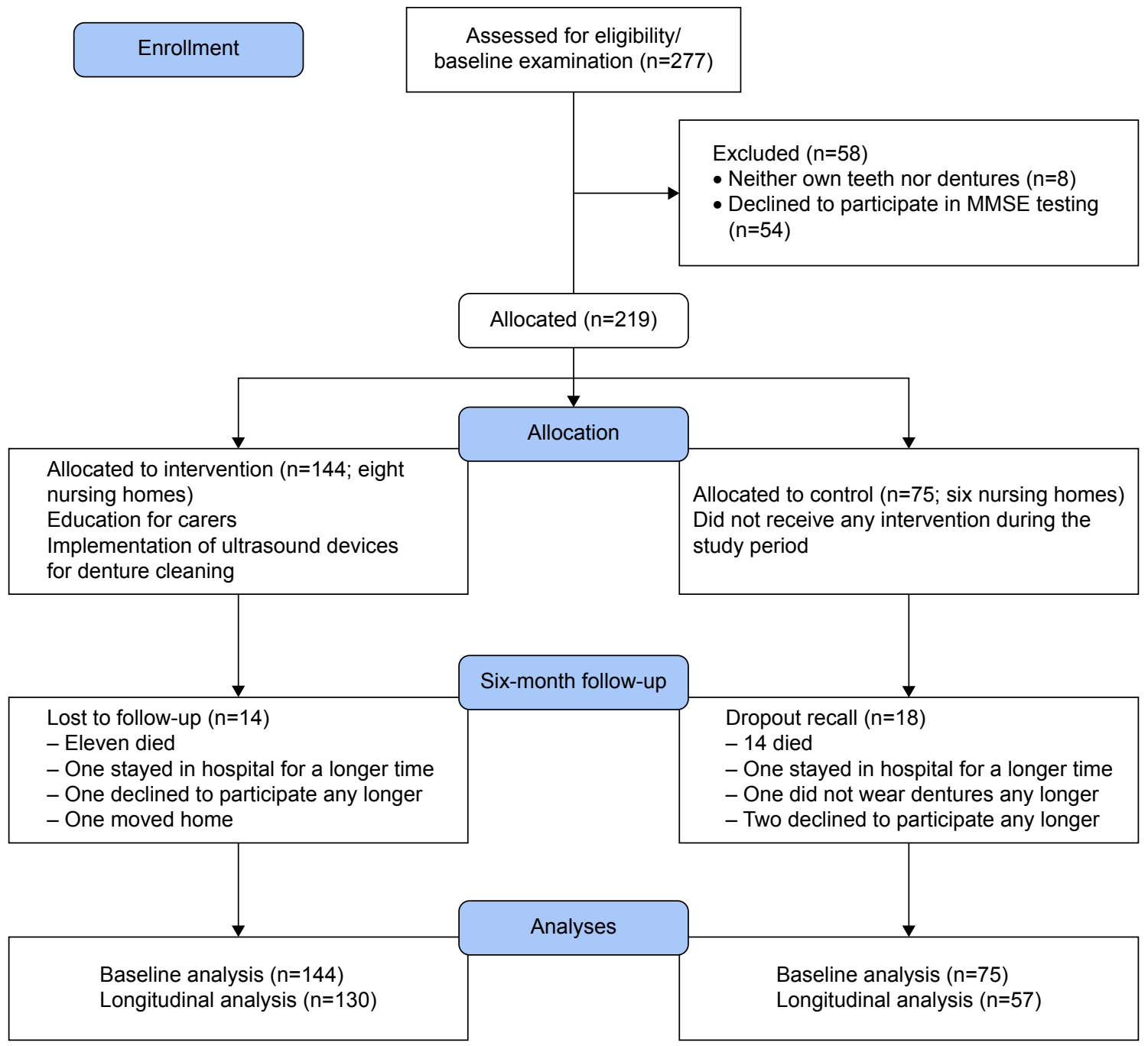

Figure I Participants' flowchart (modified according to CONSORT guidelines). Abbreviation: MMSE, Mini-Mental State Examination.

join the research team until the baseline examinations and investigated, therefore, fewer participants in this wave. The cognitive state was evaluated and documented by four psychologists trained in geriatric assessments by investigating patients attending the memory clinic of the University of Heidelberg (head: Prof Dr Johannes Schröder, MD, PhD). Additionally, demographic data such as age, sex, diseases and permanent medications were collected from the care documentation and the medical records.

\section{Dental target variables}

To evaluate oral hygiene, the Plaque Control Record (PCR) ${ }^{23}$ and the Gingival Bleeding Index $(\mathrm{GBI})^{24}$ were used. Both indices allot a calculation of the quotient of test-positive tooth surfaces and all surfaces (mesial, buccal, distal, palatal/lingual), resulting in a percentage value $(0 \%-100 \%)$. For PCR, all tooth surfaces were tinted with a plaque indicator solution (Mira-2-Ton; Hager \& Werken, Duisburg, Germany). Participants were then asked to rinse their mouth with water, followed by enumeration of positive tooth sites. For the determination of GBI, a periodontal probe was used (CPC11.5; Hu Friedy, Tutlingen, Germany). The probe was gently inserted and slid through the mesial, buccal, distal and palatal/lingual gingival sulcus of each tooth. After approximately ten seconds, bleeding sites were counted and divided by the total number of available tooth surfaces. The Community Periodontal Index of Treatment Needs (CPITN) was administered with the same probe. ${ }^{25}$ Again, the probe was inserted in the gingival sulcus of each tooth with a controlled force of 20p. Using the CPITN, five codes are 
possible for each of the up to six sextants; these codes give information on periodontal health condition; code 0 mirrors healthy conditions, codes 1 and 2 gingivitis, and codes 3 and 4 moderate and severe periodontitis, respectively. Denture hygiene was administered using the Denture Hygiene Index (DHI) ${ }^{26}$ For the evaluation of DHI, dentures were tinted with a plaque indicator (Plaque Test; Ivoclar Vivadent, Schaan, Liechtenstein), rinsed with water and evaluated for plaque-positive sites by illumination of a polymerization lamp (Bluephase; Ivoclar Vivadent). Plaque-positive areas were separately counted for each denture and divided by ten (total possible sites) to give a score, which could range from $0 \%$ to $100 \%$. Compare Zenthöfer et al. ${ }^{13}$

\section{Assessment of dementia and care dependency}

Cognitive state was determined using Mini-Mental State Examination (MMSE). All participants were asked to solve 30 little exercises. These exercises covered the dimensions orientation, short-term memory, arithmetic tasks, language use and comprehension and basic motor skills. Using MMSE, the score of completed exercises was 1 point and of failed exercises was 0 point. Therefore, the maximum score was 30 points. Scores $>20$ points are indicative of more or less normal cognitive functioning, while lower scores hint dementia. ${ }^{27}$ For analytic purposes, the MMSE cut-off value of $\leq 20$ was used for allocation of dementia and non-dementia groups. ${ }^{27}$

To assess the level of care needed, the Barthel Index (BI) was administered by qualified caregivers in the corresponding nursing homes. This assessment method was recommended in a previous study.$^{28} \mathrm{BI}$ allows evaluation of the functional capacity of older people and is therefore broadly established in epidemiologic studies on older communities. In the evaluation of functional capacity (activities of daily living), a score of 0 is indicative of total dependence, whereas a score of 100 reflects total independence. ${ }^{29}$

\section{Interventions}

Several interventions were offered to the intervention homes targeting residents and staff as well as the structure and the processes of nursing homes.

The dental interventions featured both a two-day comprehensive education program for the carers and the implementation of ultrasound baths for denture cleaning. For the educational part of the interventions, as many carers as possible were targeted to participate in the lectures. Therefore, the lectures were offered twice for each nursing home. Finally, one carer from each ward of a nursing home participated at minimum in the education program. A total of 87 caregivers passed the complete training. To impart knowledge, a standardized PowerPoint presentation was used. Education on age-related changes and pathologies of the oral cavity and a standardized estimation tool of oral conditions were provided. Carers were taught feasible teeth brushing techniques, handling of interdental space brushes and mouth rinses. Furthermore, a care movie, produced and offered by the dental association of Baden-Württemberg, Germany, was shown to the attendees. To improve the estimations of the caregivers concerning oral health conditions, a validated assessment tool was introduced to the attendees; Revised Oral Assessment Guide. ${ }^{30}$ In addition, all carers were trained in the handling of different kinds of removable dentures using demonstration models. In order to make an attempt in improving denture hygiene for all intervention homes, two ultrasound baths were each supplied (Sonorex Super RK 31H; Bandelin GmbH, Berlin, Germany); the caregivers were trained in the autonomous use of the baths during the study period. In terms of safety, it was recommended that the carers only use soapy water for the ultrasonic cleaning of the dentures. To fulfill hygienic requirements, resin molds were supplied to each denture wearer to guarantee a safe cleaning process in the bath. The second part of the interventions was of practical nature. Carers were asked to recommend seniors with whom they had problems in care routine. First, the caregivers estimated the oral health by the use of the Revised Oral Assessment Guide (ROAG); second, they took out dentures (if applicable) and cleaned dentures and natural residual teeth by themselves under the supervision of a study dentist. The dentist gave feedback and advice in all exercises. Finally, the contents of the lectures were handed out to all attendees of the homes on CD-ROM and also in a print version. In addition, 25 professional carers in leading positions of all participating intervention homes were trained as multipliers in order to provide a communication training with the special module "communication with physicians" to their colleagues. The special elements of this module were communication strategies for interaction between physicians and medical specialists engaged in nursing home care and professional caregivers. The training included exercises for structured information flow even with dentists. ${ }^{31}$

\section{Statistical analysis}

All data analyses were carried out with the aid of Statistical Package of the Social Sciences 19.0 (IBM Corporation, Armonk, NY, USA). The dental target variables PCR, GBI, CPITN and DHI were compared between intervention 
and control groups, between participants with and without dementia and between baseline and follow-up investigations by the use of independent $t$-tests. In addition to bivariate analysis, multivariate regression models for the changes in the dental target variables between baseline and follow-up (dependent variables) were compiled and confounded with age, sex, dementia/non-dementia and the level of dependency (independent variables). Local statistical significance was observed at $\alpha<0.05$.

\section{Results}

Of the 277 residents initially enrolled in the study, 54 participants declined to undergo the MMSE and eight participants had neither their own teeth nor dentures (necessary for the target variables). Therefore, 219 complete datasets were available for baseline analysis. During the six-month study period, a further 32 participants were lost to follow-up (response: 85.4\%). Thus, complete data of 187 participants were available for longitudinal analyses (Figure 1). At baseline, 150 participants $(68.5 \%)$ were women. The mean (SD) age of participants was 83.1 years (9.0). Of the control group, 48 and 27 participants lived in urban and rural nursing homes, respectively (intervention group: 94 urban participants/50 rural participants, $P=0.483$ ). The study population suffered in mean (SD) from 3.4 (2.2) chronic medical conditions and permanently took 6.5 (3.4) drugs. The mean (SD) BI among the participants was 49.5 (29.6); the mean (SD) MMSE score was 15.8 (9.1). Approximately $70 \%$ of the participants wore any kind of removable dentures for rehabilitation of at least one jaw. Approximately $40 \%$ of the sample was completely edentulous. The mean (SD) value for the number of remaining teeth among the sample was 7 (8.4). At baseline examination in the intervention and control groups, the mean (SD) PCR was found to be 85.7 (18.0) and 85.0 (21.9), the mean (SD) GBI 49.3 (26.6) and 55.8 (30.4), the mean (SD) DHI 84.5 (15.4) and 82.5 (15.5) and the mean (SD) CPITN 3.0 (0.7) and 2.9 (0.7), respectively. Cross-sectional participants' characteristics including the abovementioned dental target variables were comparable in the intervention and control groups $(P>0.05)$ with the exception of medication $(P<0.05)$. With regard to people with and without dementia, the mean CPITN scores and the number of natural teeth were different at baseline $(P<0.05)$. More detailed baseline characteristics are presented elsewhere. ${ }^{13}$ At follow-up examination after six months in the intervention group, a mean (SD) PCR value of 70.1 (26.1), a mean (SD) GBI value of 40.2 (29.3), a mean (SD) DHI value of 57.8 (27.9) and a mean (SD) CPITN value of $2.9(0.6)$ were observed, indicating significant improvements in PCR and DHI $(P<0.001)$. Significant improvements were found in people with and without dementia compared to the control group $(P<0.05)$. In the control group, no significant changes in the dental indices have been found $(P>0.05)$. For detailed bivariate analyses, see Table 1.

The multivariate regression analysis of effects on changes in PCR (Table 2), GBI (Table 3), DHI (Table 4) and CPITN (Table 5) revealed that worse baseline conditions were an indicator for more substantial improvements in the oral health indices in each model $(P<0.001)$. Furthermore, being in the intervention group was associated with significant improvements in PCR $(P=0.002)$ and DHI $(P<0.001)$. The site of the nursing home independently affected the improvements in PCR with less improvement in participants living in urban homes $(P=0.001)$. DHI significantly improved in more care-dependent subjects compared to those who needed less help by the caregivers $(P<0.001)$. Consistently, age, sex, number of chronic diseases, permanent medications and prevalence of dementia had no independent effect on the improvement in dental indices $(P>0.05)$, albeit a clear trend to slightly lower improvements in PCR was observed in participants with dementia $(P=0.053)$.

\section{Discussion}

The results of this study indicated worse oral health conditions compared to previous cross-sectional studies in

Table I Target variables for participants with and without dementia in the intervention and control groups at baseline and follow-up after 6 months

\begin{tabular}{llllll}
\hline Variable & \multicolumn{3}{l}{ Baseline } & & \multicolumn{3}{l}{ Follow-up } \\
\cline { 2 - 3 } \cline { 5 - 6 } & Intervention & Control & & Intervention & Control \\
\hline PCR, mean (SD) & $85.7(18.0)^{\mathrm{a}}$ & $85.0(21.9)$ & $70.1(26.1)^{\mathrm{a}}$ & $87.9(15.8)$ \\
Dementia & $89.8(11.9)^{\mathrm{b}}$ & $84.1(23.7)$ & $77.0(24.6)^{\mathrm{b}}$ & $89.1(14.7)$ \\
Non-dementia & $81.6(21.9)^{\mathrm{c}}$ & $87.3(17.3)$ & $63.2(26.1)^{\mathrm{c}}$ & $83.9(19.6)$ \\
GBI, mean (SD) & $49.3(26.6)$ & $55.8(30.4)$ & $40.2(29.3)$ & $51.3(28.3)$ \\
Dementia & $51.2(25.5)$ & $57.0(30.0)$ & $44.6(30.1)$ & $51.5(28.1)$ \\
Non-dementia & $47.4(27.9)$ & $52.8(32.2)$ & $35.8(28.2)$ & $50.4(30.7)$ \\
DHI, mean (SD) & $84.5(15.4)^{\mathrm{a}}$ & $82.5(15.5)$ & $57.8(27.9)^{\mathrm{a}}$ & $77.6(22.6)$ \\
Dementia & $86.3(15.6)^{\mathrm{a}}$ & $84.3(13.6)$ & $52.6(30.3)^{\mathrm{a}}$ & $79.2(21.9)$ \\
Non-dementia & $82.2(15.0)^{\mathrm{a}}$ & $76.3(20.4)$ & $63.4(24.2)^{\mathrm{a}}$ & $70.6(25.8)$ \\
CPITN, mean & $3.0(0.7)$ & $2.8(0.7)$ & $2.9(0.6)$ & $2.8(0.8)$ \\
(SD) & & & & \\
Dementia & $3.3(0.6)$ & $2.8(0.8)$ & $3.1(0.6)$ & $2.8(0.9)$ \\
Non-dementia & $2.8(0.6)$ & $2.7(0.6)$ & $2.8(0.6)$ & $2.7(0.7)$ \\
Number of & $7.4(8.6)$ & $6.4(8.1)$ & $7.0(8.6)$ & $5.8(8.1)$ \\
teeth, mean (SD) & & & & \\
Dementia & $6.3(8.0)$ & $6.2(8.5)$ & $6.2(7.9)$ & $5.9(7.9)$ \\
Non-dementia & $8.9(9.1)$ & $7.1(7.1)^{\mathrm{a}}$ & $8.1(8.4)$ & $5.3(6.8)^{\mathrm{a}}$ \\
\hline
\end{tabular}

Notes: Unpaired $t$-test: ${ }^{\mathrm{P} P}<0.001,{ }^{\mathrm{b} P}=0.002,{ }^{\mathrm{c} P}=0.001$.

Abbreviations: PCR, Plaque Control Record; GBI, Gingival Bleeding Index; CPITN, Community Periodontal Index of Treatment Needs; DHI, Denture Hygiene Index. 
Table 2 Linear regression model for changes in PCR as a dependent variable $(n=1 \mid 8)$

\begin{tabular}{llll}
\hline Effect & Regression & $\mathbf{9 5 \%} \mathbf{C l}$ & $P$-value \\
\hline Age & -0.2 & $-0.6,0.3$ & 0.424 \\
Female & 6.7 & $-2.7,16.2$ & 0.162 \\
Diseases & 0.3 & $-1.5,2.2$ & 0.718 \\
Medication & -0.1 & $-1.3,1.2$ & 0.918 \\
Dementia & 8.5 & $-0.1,17.2$ & 0.053 \\
Care dependency & 0.1 & $-0.1,0.2$ & 0.453 \\
Intervention & -13.7 & $-22.1,-5.2$ & $\mathbf{0 . 0 0 2}$ \\
Rural & -14.4 & $-22.7,6.1$ & $\mathbf{0 . 0 0 1}$ \\
Baseline PCR & -0.6 & $-0.8,-0.3$ & $\mathbf{0 . 0 0 1}$ \\
\hline
\end{tabular}

Notes: Negative values are indicative for improvements. $R^{2}=0.399$. Data in bold indicates statistical significance.

Abbreviation: PCR, Plaque Control Record.

non-demented, less care-dependent older people. ${ }^{1,3-5}$ This recent study, however, also proved the effectiveness of dental education for carers and implementation of ultrasound devices in order to improve oral health and denture hygiene of care-dependent and/or older people with dementia in nursing homes. During the six-month study period, oral hygiene as measured by PCR and denture hygiene (DHI) significantly improved in the intervention group, whereas no effects could be observed in the control group. These results are comparable to those found in a previous intervention study, albeit the participants in our recent study were more cognitively impaired. ${ }^{22}$ In this context, the improvements in denture hygiene have to be evaluated as especially relevant. This study has demonstrated that the introduction of ultrasound baths is a very successful measure for sustainable improvement in denture hygiene. It should also be stressed that primarily high-maintenance older people (low BI) profited from the denture cleanings. This is important for two reasons: on the one hand, previous research revealed strong correlations between denture plaque and the incidence of pneumonia; on the other hand, care-dependent multi-morbid older people

Table 3 Linear regression model for changes in $\mathrm{GBI}$ as a dependent variable $(\mathrm{n}=\mathrm{I} \mid 8)$

\begin{tabular}{llll}
\hline Effect & Regression & $\mathbf{9 5 \%} \mathbf{~ C l}$ & P-value \\
\hline Age & -0.1 & $-0.7,0.6$ & 0.820 \\
Female & 3.2 & $-16.2,9.8$ & 0.628 \\
Diseases & -0.5 & $-3.1,2.0$ & 0.672 \\
Medication & -0.2 & $-1.9,1.5$ & 0.845 \\
Dementia & 3.4 & $-8.5,15.3$ & 0.572 \\
Care dependency & 0.1 & $-0.1,0.3$ & 0.166 \\
Intervention & -8.2 & $-19.8,3.5$ & 0.169 \\
Rural & -7.4 & $-19.0,4.2$ & 0.208 \\
Baseline GBI & -0.7 & $-0.9,-0.5$ & $\mathbf{0 . 0 0 I}$ \\
\hline
\end{tabular}

Notes: Negative values are indicative for improvements. $R^{2}=0.367$. Data in bold indicates statistical significance.

Abbreviation: $\mathrm{GBI}$, Gingival Bleeding Index.
Table 4 Linear regression model for changes in $\mathrm{DHI}$ as a dependent variable $(n=138)$

\begin{tabular}{llll}
\hline Effect & Regression & $\mathbf{9 5 \%} \mathbf{C l}$ & $P$-value \\
\hline Age & 0.1 & $-0.5,0.7$ & 0.810 \\
Female & 1.0 & $-9.0,11.0$ & 0.847 \\
Diseases & -0.8 & $-2.6,0.9$ & 0.340 \\
Medication & 0.0 & $-1.2,1.1$ & 0.982 \\
Dementia & -3.4 & $-12.7,5.9$ & 0.470 \\
Care dependency & -0.3 & $-0.4,0.1$ & $\mathbf{0 . 0 0 I}$ \\
Intervention & -20.1 & $-29.5,-10.6$ & $\mathbf{0 . 0 0 I}$ \\
Rural & 6.9 & $-1.4,15.1$ & 0.100 \\
Baseline DHI & -0.5 & $-0.8,-0.2$ & $\mathbf{0 . 0 0 I}$ \\
\hline
\end{tabular}

Notes: Negative values are indicative for improvements. $R^{2}=0.318$. Data in bold indicates statistical significance.

Abbreviation: DHI, Denture Hygiene Index.

are specifically prone to diseases such as bacteremia because of their reduced immune status. ${ }^{15,16,20}$ As this investigation highlights, this problem can be overcome with a simple measure. Moreover, a substantial reduction in dental plaque accumulation has been observed during the study period; dental plaque is also capable of provoking pneumonia and a reduction is able to reduce this risk and vice versa. This specific study outcome has to be evaluated at that positive because professional teeth cleaning - which might have had a considerable effect on the potential to improve - was not part of the interventions. Therefore, in some cases, it was securely not possible for the trained and motivated carers to remove calculus, which might have limited larger improvements. This confounder should be the topic of further research. However, the aim of this recent study was to test concepts to improve oral health, which are easily applicable in the daily care routine. In analysis of GBI and CPITN, no significant improvements could be measured over the study period, albeit a substantial trend to improvement was observed. One should keep in mind that GBI can be affected by anticoagulative medications, which were present in $\sim 40 \%$

Table 5 Linear regression model for changes in CPITN as a dependent variable $(n=I \mid 8)$

\begin{tabular}{llll}
\hline Effect & Regression & $\mathbf{9 5 \%} \mathbf{C l}$ & $\boldsymbol{P}$-value \\
\hline Age & 0.0 & $0.0,0.0$ & 0.978 \\
Female & -0.2 & $-0.4,0.1$ & 0.237 \\
Diseases & 0.0 & $-0.1,0.0$ & 0.672 \\
Medication & 0.0 & $0.0,0.1$ & 0.151 \\
Dementia & 0.0 & $-0.2,0.3$ & 0.803 \\
Care dependency & 0.0 & $0.0,0.0$ & 0.941 \\
Intervention & 0.0 & $-0.2,0.3$ & 0.869 \\
Rural & 0.0 & $-0.2,0.3$ & 0.887 \\
Baseline CPITN & -0.5 & $-0.7,-0.3$ & $\mathbf{0 . 0 0 I}$ \\
\hline
\end{tabular}

Notes: Negative values are indicative for improvements. $R^{2}=0.263$. Data in bold indicates statistical significance.

Abbreviation: CPITN, Community Periodontal Index of Treatment Needs. 
of the participants. ${ }^{13}$ Furthermore, $89 \%$ of the participants had periodontitis in at least one sextant (see CPITN). Improvement in the severity of periodontitis would have required a systematic periodontitis therapy. Therefore, this outcome is as expected. ${ }^{32}$

In general, it should be considered that the results of this study would have substantially been affected by the transfer of caregivers' improved knowledge in the care routine. The interventions probably did not change carers' attitudes concerning oral hygiene area wide. Moreover, lack of time was probably still a limitation for adequate oral hygiene procedures. ${ }^{7}$ This effect seemed to be more evident in larger urban long-term care homes (less improvement in PCR compared to rural homes; $P<0.001$ ). A further limitation of the study outcome might have been care-resistant, affective and uncooperative behavior highly prevalent in seniors suffering from dementia. ${ }^{9}$ Our sample included $\sim 60 \%$ participants with more or less severe dementia. It should also be borne in mind that cognitive and motor function can decrease over time, which can be associated with worsening of oral conditions. ${ }^{19}$ The most positive aspect of this study was that it was able to reduce plaque accumulation and denture plaque likewise in participants with and without dementia. To this end and albeit all constraints associated with nursing aspects, one might highlight that the investigated interventions are efficient over a clinically relevant period of time. In general, it should be considered that intra-individual baseline conditions (size of the dental indices) were significantly related to changes in oral health as well as denture hygiene with higher improvements in subjects with worse baseline conditions.

\section{Study limitations}

The results of this study could be biased due to the exclusion of 54 participants refusing to undergo MMSE tests. However, this variable was needed for clarification of the research questions. One should also consider that neither the carers' knowledge nor their attitudes concerning oral health were considered. This, however, is a limitation of this study as it might have an impact on the efficacy of the interventions.

\section{Conclusion}

Within the limitations of this study, carers' education improves oral health of care-dependent nursing home residents with and without dementia over a clinically relevant period of time. The implementation of ultrasound baths for denture cleaning is a simple but effective measure to improve denture hygiene in both institutionalized older people and seniors with dementia and in severe need of care. Improved communication between carers and residents as well as relatives and health care professionals may be helpful and improves residents' (dental) care quality. However, longer study periods are needed to clarify if these positive effects are stable in the long term or whether renewal of the interventions is necessary.

\section{Acknowledgments}

We are grateful to Sozialministerium Baden-Württemberg (Ministry of Social Affairs) for financial support of the study. We thank all participants of this study for their patience during the examinations. We are grateful to Laura Minnich for English language revision. Furthermore, we thank Lina Gorenc, Nadja Urbanowitsch, Sabrina Navratil and Petra Wetzel for assessment of MMSEs.

\section{Disclosure}

The authors report no conflicts of interest in this work.

\section{References}

1. Furuta M, Komiya-Nonaka M, Akifusa S, et al. Interrelationship of oral health status, swallowing function, nutritional status, and cognitive ability with activities of daily living in Japanese elderly people receiving home care services due to physical disabilities. Community Dent Oral Epidemiol. 2013;41(2):173-181.

2. Komulainen K, Ylöstalo P, Syrjälä AM, et al. Oral Health intervention among community-dwelling older people: a randomised 2-year intervention study. Gerodontology. 2015;32(1):62-72.

3. Chalmers JM, Carter KD, Spencer AJ. Caries incidence and increments in Adelaide nursing home residents. Spec Care Dentist. 2005;25(2): 96-105.

4. Matthews DC, Clovis JB, Brillant MG, et al. Oral health status of longterm care residents-a vulnerable population. J Can Dent Assoc. 2012; $78(3): \mathrm{c} 3$

5. McMillan AS, Wong MCM, Lo ECM, Allen F. The impact of oral disease among the institutionalized and non-institutionalized elderly in Hong Kong. J Oral Rehabil. 2003;30(1):46-54.

6. Fiske J, Griffiths J, Jamieson R, Manger D. Guidelines for oral health care for long-stay patients and residents. Gerodontology. 2000;17(1): $55-64$

7. Adams R. Qualified nurses lack adequate knowledge related to oral health, resulting in inadequate oral care of patients on medical wards. J Adv Nurs. 1999;24(3):552-560.

8. Nitschke I, Müller F, Hopfenmüller W. The uptake of dental services by elderly Germans. Gerodontology. 2001;18(2):114-120.

9. Mahoney EK, Hurley AC, Volicer L, et al. Development and testing of the resistiveness to Care Scale. Res Nurs Health. 1999;22(1):27-38.

10. Rejnefelt I, Andersson P, Renvert S. Oral health status in individuals with dementia living in special facilities. Int J Dent Hyg. 2006;4(2): 67-71.

11. Syrjälä AM, Ylöstalo $P$, Ruoppi $P$, et al. Dementia and oral health among subjects aged 75 years or older. Gerodontology. 2012;29(1) 36-42.

12. Zenthöfer A, Schröder J, Cabrera T, Rammelsberg P, Hassel AJ. Comparison of oral health among older people with and without dementia. Community Dent Health . 2014;31(1):1-5.

13. Zenthöfer A, Baumgart D, Cabrera T, et al. Poor dental hygiene and periodontal health in nursing home residents with dementia: an observational study. Odontology. Epub 2016 May 9.

14. Dörfer CE, Becher H, Ziegler CM, et al. The association of gingivitis and periodontitis with ischemic stroke. J Clin Periodontol. 2004;31(5): 396-401. 
15. Scannapieco FA. Position paper of the American Academy of Periodontology: periodontal disease as a potential risk factor for systemic diseases. J Periodontol. 1998;69(7):841-850.

16. Scannapieco FA. Pneumonia in nonambulatory patients. The role of oral bacteria and oral hygiene. Am Dent Assoc. 2006;137(1):21-25.

17. Nicol R, Petrina Sweeney M, McHugh S, Bagg J. Effectiveness of health care worker training on the oral health of elderly residents of nursing homes. Community Dent Oral Epidemiol. 2005;33(2):115-124.

18. Ribeiro DG, Pavarina AC, Giampaolo ET, Machado AL, Jorge JH, Garcia PP. Effect of oral hygiene education and motivation on removable partial denture wearers: longitudinal study. Gerodontology. 2009;26(2): $150-156$.

19. Zenthöfer A, Dieke R, Dieke A, Wege KC, Rammelsberg P, Hassel AJ. Improving oral hygiene in the long-term care of the elderly - a RCT. Community Dent Oral Epidemiol. 2013;41(3):261-268.

20. Adachi M, Ishihara K, Abe S, Okuda K, Ishikawa T. Effect of professional oral health care on the elderly living in nursing homes. Oral Surg Oral Med Oral Pathol Oral Radiol Endod. 2002;94(2):191-195.

21. De Visschere L, de Baat C, Schols JM, Deschepper E, Vanobbergen J. Evaluation of the implementation of an 'oral hygiene protocol' in nursing homes: a 5-year longitudinal study. Community Dent Oral Epidemiol. 2011;39(5):416-425.

22. van der Putten GJ, Mulder J, de Baat C, De Visschere LM, Vanobbergen JN, Schols JM. Effectiveness of supervised implementation of an oral health care guideline in care homes; a single-blinded cluster randomized controlled trial. Clin Oral Investig. 2013;17(4): 1143-1153.

23. O'Leary TJ, Drake RB, Naylor JE. The plaque control record. J Periodontol. 1972;43(1):38-44.
24. Ainamo J, Bay I. Problems and proposals for recording gingivitis and plaque. Int Dent J. 1975;25(4):229-235.

25. Ainamo J, Barmes D, Beagrie G, Cutress T, Martin J, Sardo-Infirri J. Development of the World Health Organization (WHO) community periodontal index of treatment needs (CPITN). Int Dent J. 1982;32(3): 281-291.

26. Wefers KP. Der "denture hygiene index". Dent Forum. 1999;1(1): 3-15.

27. Folstein MF, Folstein SE, McHugh PR. "Mini-mental state". A practical method for grading the cognitive state of patients for the clinician. J Psychiatr Res. 1975;12(3):189-198.

28. Wade DT, Collin C. The Barthel ADL Index: a standard measure of physical disability? Int Disabil Stud. 1988;10(2):64-67.

29. Mahoney FI, Barthel DW. Functional evaluation: the Barthel index. Md State Med J. 1965;14(1):61-65.

30. Hassel AJ, Leisen J, Rolko C, Rexroth W, Ohlmann B, Rammelsberg P. Clinical assessment of oral health between physician and dentist - a pilot study on inter-examiner reliability. Z Gerontol Geriatr. 2008;41(2): $132-138$.

31. Meyer-Kühling I, Wendelstein B, Pantel J, Specht-Leible N, Zenthöfer A, Schröder J. Communication training MultiTANDEMplus - a contribution to improve communication between professional caregivers and physicians. Pflege. 2015;28(5):277-285.

32. Imrey PB, Chilton NW, Pihlstrom BL, et al. Proposed guidelines for American Dental Association acceptance of products for professional, non-surgical treatment of adult periodontitis. Task force on design and analysis in dental and oral research. J Periodontal Res. 1994;29(5): 348-360.
Clinical Interventions in Aging

\section{Publish your work in this journal}

Clinical Interventions in Aging is an international, peer-reviewed journal focusing on evidence-based reports on the value or lack thereof of treatments intended to prevent or delay the onset of maladaptive correlates of aging in human beings. This journal is indexed on PubMed Central, MedLine,

\section{Dovepress}

CAS, Scopus and the Elsevier Bibliographic databases. The manuscript management system is completely online and includes a very quick and fair peer-review system, which is all easy to use. Visit http://www.dovepress. com/testimonials.php to read real quotes from published authors. 